\title{
Kongressbericht vom 3. Brustkrebskongress Köln und Niederrhein
}

\author{
Rachel Würstlein ${ }^{\mathrm{a}}$ Oleg Gluz $^{\mathrm{b}}$ Friedrich Wolffc, \\ im Namen der wissenschaftlichen Leitung BKK 2010 \\ a Brustzentrum, Universitätsfrauenklinik Köln, \\ ${ }^{\mathrm{b}}$ Brustzentrum Niederrhein, Evangelisches Krankenhaus Bethesda, Mönchengladbach, \\ ${ }^{\circ}$ Frauenklinik, Krankenhaus Holweide, Kliniken der Stadt Köln, Germany
}

Am 09. Januar 2010 fand als erste Brustkrebs-Veranstaltung in NRW im neuen Jahr der 3. Brustkrebskongress Köln und Niederrhein statt. Die Organisatoren (Abb. 1), das Brustzentrum Niederrhein (Leitung Frau Pof. Nitz, Mitorganisator: Herr Dr. Gluz), das Brustzentrum der Kliniken der Stadt Köln Krankenhaus Holweide (Leitung Herr Prof. Wolff; bis 12/09 Frau Dr. Fischer) sowie das Brustzentrum Köln-Frechen, Standort Universitätsklinik Köln (Leitung Frau Prof. Harbeck, Herr PD Dr. Warm; Direktor der UFK Herr Prof. Mallmann) konnten trotz Schneesturm Daisy über 300 Teilnehmer in Köln begrüßen. Unterstützt wurde der Kongress durch eine große, breit gefächerte Industrieausstellung sowie die Zusammenarbeit mit HR und C Medical, München. Der diesjährige Kongress der Kölner Brustzentren, erstmals mit Beteiligung des Brustzentrums Niederrhein und mit gleichzeitigem Post-San-Antonio-Update war damit der bisher größte in den vergangenen drei Jahren. Entsprechend stolz zeigte sich in ihrer Begrüßungsrede auch die Bürgermeisterin der Stadt Köln, Frau Scho-Antwerpes, auf die optimale Betreuung von Patientinnen mit Brusterkrankungen in der Region.

Highlight der Veranstaltung war die Key-Note-Lecture von Frau Prof. Martine Piccart (Institut Jules Bordet, Brüssel, Abb. 2) mit dem Thema «The new strategy oft the BIG to accelerate tailored oncology in early breast cancer». Hier zeigte Frau Piccart in einem sehr persönlichen Vortrag den Werdegang der BIGStudiengruppe in Europa auf. Die Bedeutung des Zusammenschlusses von Studiengruppen, des neuen Denkens weg von «one strategy fits all» sowie die Integration von translationaler Forschung in die klinischen Studien sind die drei entscheidenden Schritte für moderne onkologische Studienkonzepte insbesondere in der (neo)adjuvanten Situation. Frau Prof. Piccart hatte die Ehre, im Dezember 2009 die William L. McGuire Memorial Lecture in San Antonio zu halten - diese aktuellen Ausführungen zur internationalen Brustkrebsforschung konnten die Kongressteilnehmer dank der guten wissenschaftlichen Kooperationen der Veranstalter mit Frau Piccart und der BIG in Köln nun zeitnah verfolgen.

Frau Prof. Nitz stellte die 15-jährige Entwicklung der Westdeutschen Studiengruppe (WSG) anhand des Beispiels $«<\mathrm{Maß-}$ geschneiderte> Chemotherapien im Zeitalter von Gensignaturen» dar. Die WSG spielt national und international eine wichtige Rolle bei der Studienentwicklung in der adjuvanten und nun auch metastasierten Situation. Die Teilnehmer konnten die Entwicklungsschritte eines Studienprotokolls an mehreren erfolgreich abgeschlossenen Studienbeispielen nachvollziehen. Besonders wurden die Studien Plan B und MINDACT im Kontext der individualisierten Chemotherapie beim Mammakarzinom hervorgehoben. In der Plan-B-Studie wird die Wertigkeit des durch die WSG etablierten Standards von EC-Doc gegen das anthracyclinfreie Regime 6 Zyklen TC bei HER2 negativem primären Mammakarzinom in der adjuvanten Situation geprüft. Bei Patientinnen mit 0 bis 3 befallenen Lymphknoten und hormonrezeptorpositiver Erkrankung wird hier außerdem zur Vermeidung von Überbehandlung durch Chemotherapie der Multigentest Oncotype DX eingesetzt (weitere Informationen zu den Studienkonzepten der WSG: www.wsg-online.com).

Im ersten Teil der Veranstaltung ging es um den Umgang mit Vorläuferläsionen aus dem Screening mit Schwerpunkt der nicht-invasiven Mammatumoren. Herr PD Dr. Gossmann (Kliniken der Stadt Köln, Krankenhaus Merheim, Radiologie) erklärte neue Vorgehensweisen bei Mammadiagnostik und minimalinvasiven Mammabiopsien, insbesondere unter Berücksichtigung der Bedeutung von Mikrokalzifikationen. Aus Sicht des Pathologen stellte Herr Dr. Pelz (Institut für Pathologie, Viersen) die sogenannten B3-Läsionen dar. Läsionen mit dem Risiko eines assoziierten DCIS oder invasiven Karzinoms wie ADH, FEA und LN sowie inhomogene Läsionen mit SamplingRisiko (Radiäre Narbe, Papillom, Phylloidestumor) wurden in ihrer klinischen Bedeutung mit den Teilnehmern und den Vertretern der Brustzentren diskutiert. Diese Diskussion wurde durch die Darstellung der klinischen Entscheidungsfindung (Herr Prof. Blohmer, St. Gertrauden Krankenhaus Berlin) vertieft. Auch die Arbeitsgemeinschaft gynäkologische Onkologie (AGO) berücksichtigt diese Läsionen in den neuen Leitlinien (www.ago-online.de). Die Notwendigkeit der individuellen Diskussion mit der Patientin wurde von allen Brustzentren bestätigt.

\section{KARGER}

Fax +497614520714

Information@Karger.de

www.karger.com
(C) 2010 S. Karger GmbH, Freiburg

Accessible online at:

www.karger.com/brc
Dr. Rachel Würstlein

Brustzentrum, Universitätsfrauenklinik Köln

Kerpener Str. 34, 50931 Köln, Germany

Tel. +49 221 478-87743, Fax -86546

rachel.wuerstlein@uk-koeln.de 


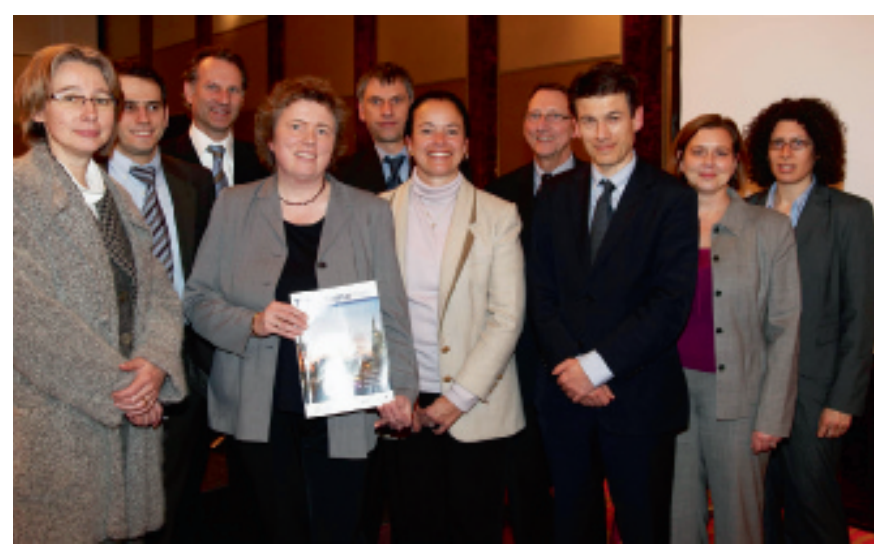

Abb. 1. Das Organisationsteam des BKK 2010. Von links nach rechts: Prof. Dr. Ulrike Nitz, Dr. Oleg Gluz, Dr. Thomas Winter, Prof. Dr. Nadia Harbeck, PD Dr. Mathias Warm, Prof. Dr. Martine Piccart, Prof. Dr. Friedrich Wolff, Prof. Dr. Peter Mallmann, Dr. Rachel Würstlein, Dr. Cordula Moers. ${ }^{\oplus}$ medfoto, Dorothea Hensen.

Aufgrund der Aktualität in der Region folgte dann ein Update zum Thema der intraoperativen Strahlentherapie (IORT) aus der Sicht des Strahlentherapeuten (Dr. Altmann, MVZ Mönchengladbach) sowie des Klinikers, Herrn PD Dr. Friedrichs (Mammazentrum, Hamburg). Als Indikationen für die intraoperative Bestrahlung werden die Verkürzung der Boost-Bestrahlung bei brusterhaltender Operation, die erneute Bestrahlung nach brusterhaltender Operation eines Lokalrezidivs sowie die IORT als reine Teilbrustbestrahlung (z.B. im Rahmen der TARGITStudien) gesehen. Die verbesserte interdisziplinäre Kooperation von Diagnostiker, Operateur, Strahlentherapeut und Pathologe spielt hier eine besondere Rolle und setzt funktionierende Strukturen eines Brustzentrums voraus. Die Methode der intraoperativen Bestrahlung mittels Intrabeam ${ }^{\circledR}$ wird sowohl im Brustzentrum Niederrhein, Evangelisches Krankenhaus Bethesda Mönchengladbach, als auch im Brustzentrum Köln-Frechen, Standort Uniklinik Köln den Patientinnen angeboten.

Dem Thema optimale Versorgung vor dem Hintergrund limitierter Ressourcen widmete sich Herr Dr. Tuschen (megapharm $\mathrm{GmbH}$ ). Er stellte die Bedeutung von interdisziplinären onkologischen Netzwerken zur Versorgung onkologischer Patienten in den Vordergrund. Diese Kooperationen können durch nachgewiesene hohe Behandlungsqualität und Effizienz die Schlüsselposition in der onkologischen Versorgung einnehmen. Qualitätssicherung, interdisziplinäre Kooperation und sektorübergreifende Strukturen sind Voraussetzung für die zukünftige optimale und innovative Patientenversorgung. Als Beispiel für ein Modell sektorübergreifender Versorgung zwischen $\mathrm{Zu}$ - und Einweisern und den Brustzentren stellte Frau Dr. Würstlein stellvertretend für die drei Kölner Brustzentren (St. Elisabeth Krankenhaus Hohenlind, Kliniken der Stadt Köln, Brustzentrum Köln-Frechen, Standort Uniklinik Köln) das zusammen mit der Ärztekammer Westfalen Lippe (Leitung des Projekts: Herr Dr. Bücker-Nott, Frau Dr. Gilles) in intensiven Diskussionen mit den Niedergelassenen erarbeitete und von der ÄKZert zertifizierte Modellprojekt vor, das im Januar 2010 in Köln startet. Das Zertifikat zum Kooperationspartner der Brustzentren in NRW nach dem Kölner Modell sieht drei Bausteine vor: ein sechsteiliges in-

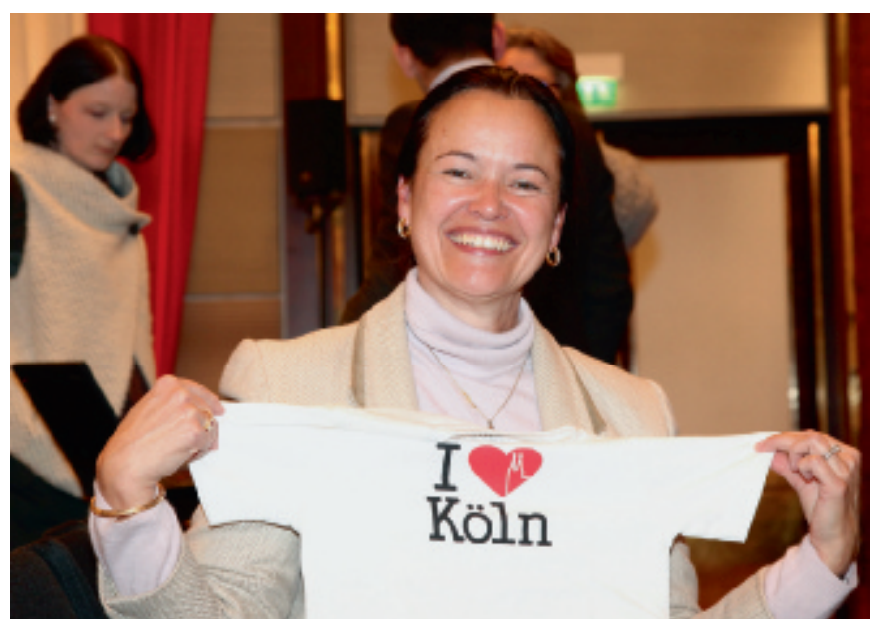

Abb. 2. Frau Prof. Piccart bekräftigt die gute Zusammenarbeit mit der Region Köln und den veranstaltenden Brustzentren. ${ }^{\circ}$ medfoto, Dorothea Hensen.

terdisziplinäres Curriculum mit Prüfung und Hospitationen im Brustzentrum, eine Zertifizierung als Kooperationspartner durch Prüfung der Schnittstellen Praxis / Brustzentrum anhand erarbeiteter Indikatoren sowie das Vorliegen eines zertifizierten Qualitäts-Management-Systems. Nähere Informationen gibt es unter www.äkwl.de oder auf der Homepage der drei Kölner Brustzentren (www.brustkrebsschwerpunkt-koeln.de, www.kliniken-koeln. de, www.hohenlind.de/fachbereiche/brustzentrum_senologie).

Das Update San Antonio 2009 mit relevanten Informationen für Klinik und Praxis stellten Herr Prof. Thomssen (Universitätsfrauenklinik Halle/Saale) für die adjuvante Situation sowie Herr Prof. Schneeweiss (Onkologische Ambulanz, Universitätsfrauenklinik Heidelberg) für die metastasierte Situation vor. Das Besondere am Kongress in San Antonio 2009 war zum einen, dass auch viele Studien mit negativen Ergebnissen dargestellt und diskutiert wurden. Zum anderen wurde intensiv diskutiert, dass durch Cross-over in den laufenden Studien zwar immer früher den Patientinnen der Zugang zu neuen Therapieoptionen eröffnet wird, dies aber die wissenschaftliche Auswertung der großen Studienprojekte erschwert. Die moderne Brustkrebsforschung gewinnt so zunehmend an Komplexität, ein Punkt, den auch Frau Piccart in ihrer Key-Note-Lecture wieder aufgriff. In Kooperation mit der American Association for Cancer Research (AACR) setzt San Antonio zunehmend auf die Relevanz der translationalen Forschung. Prognostische und prädiktive Faktoren gewinnen in der Therapieentscheidung und Individualisierung der Therapie eine zunehmende Bedeutung, ebenso die Tumorwachstumsfaktoren. Angesichts der Vielfalt neuer zielgerichteter wirksamer Moleküle im Labor und in der klinischen Entwicklung wird die Identifikation der davon profitierenden Subguppen entscheidend sein.

Mit vielen neuen Informationen versorgt gingen die Teilnehmer des diesjährigen BKK zurück in die Praxis bzw. Klinik. Wie immer bleiben praxis- und klinikrelevante Fragestellungen, so dass jetzt bereits die Planungen für den Brustkrebskongress Köln und Niederrhein Anfang 2011 anlaufen. Weitere Informationen zum diesjährigen Kongress, zu weiteren gemeinsamen Veranstaltungen sowie zum BKK 2011 finden sich auf der Homepage der veranstaltenden Brustzentren. 